\title{
Starspot activity of HD 199178
}

\section{Doppler images from 1994-2017 $\star, \star \star$}

\author{
T. Hackman ${ }^{1}$, I. Ilyin ${ }^{2}$, J. J. Lehtinen ${ }^{3,4}$, O. Kochukhov ${ }^{5}$, M. J. Käpylä ${ }^{3,4}$, N. Piskunov ${ }^{5}$, and T. Willamo ${ }^{1}$ \\ 1 Department of Physics, PO Box 64, 00014 University of Helsinki, Finland \\ e-mail: thomas .hackman@helsinki.fi \\ ${ }^{2}$ Leibniz Institute for Astrophysics Potsdam (AIP), An der Sternwarte 16, 14482 Potsdam, Germany \\ 3 Max Planck Institute for Solar System Research, Justus-von-Liebig-Weg 3, 37077 Göttingen, Germany \\ ${ }^{4}$ ReSoLVE Centre of Excellence, Department of Computer Science, Aalto University, PO Box 15400, 00076 Aalto, Finland \\ 5 Department of Physics and Astronomy, Uppsala University, Box 516, 75120 Uppsala, Sweden
}

Received 3 December 2018 / Accepted 11 March 2019

\begin{abstract}
Context. Studying the spots of late-type stars is crucial for distinguishing between the various proposed dynamo mechanisms believed to be the main cause of starspot activity. For this research it is important to collect observation time series that are long enough to unravel both long- and short-term spot evolution. Doppler imaging is a very efficient method for studying spots of stars that cannot be angularly resolved.

Aims. High-resolution spectral observations during 1994-2017 are analysed in order to reveal long- and short-term changes in the spot activity of the FK Comae-type subgiant HD 199178.

Methods. Most of the observations were collected with the Nordic Optical Telescope. The Doppler imaging temperature maps were calculated using an inversion technique based on Tikhonov regularisation and utilising multiple spectral lines.

Results. We present a unique series of 41 temperature maps spanning more than 23 years. All reliable images show a large cool spot region centred near the visible rotation pole. Some lower latitude cool features are also recovered, although the reliability of these is questionable. There is an expected anti-correlation between the mean surface temperature and the spot coverage. Using the Doppler images, we construct the equivalent of a solar butterfly diagram for HD 199178.

Conclusions. HD 199178 clearly has a long-term large and cool spot structure at the rotational pole. This spot structure dominated the spot activity during the years 1994-2017. The size and position of the structure has evolved with time, with a gradual increase during the last years. The lack of lower latitude features prevents the determination of a possible differential rotation.
\end{abstract}

Key words. stars: activity - starspots - stars: late-type - stars: imaging - stars: individual: HD 199178

\section{Introduction}

HD 199178 (also known as V1794 Cyg) is one of the original members of the FK Comae-type stars originally defined by Bopp \& Rucinski (1981). This very small group consists of stars that are single, rapidly rotating, and extremely active G-K-type subgiants or giants. The magnetic activity is observed both as strong emission from the corona, transition region, and chromosphere and as large cool spots causing rotational modulation of the brightness and photospheric absorption line profiles.

There are very few confirmed FK Comae-type stars (only three or four). The reason for this is that the class probably represents a very short evolutionary stage. Bopp \& Rucinski (1981) postulated that they are coalesced W Uma-type systems. Thus, they would be observed in the transitionary stage when they have formed a single star, but still rotate rapidly. Eventually the rapid rotation will slow down due to magnetic braking. Even

\footnotetext{
* The spectra are only available at the CDS via anonymous ftp to cdsarc.u-strasbg. fr (130.79.128.5) or via http://cdsarc . u-strasbg. fr/viz-bin/qcat?J/A+A/625/A79

$\star \star$ Based on observations made with the Nordic Optical Telescope, operated by the Nordic Optical Telescope Scientific Association at the Observatorio del Roque de los Muchachos, La Palma, Spain, of the Instituto de Astrofisica de Canarias.
}

though these stars are rare, they still provide useful information on stellar magnetic activity. It seems that most rapidly rotating latetype stars show similar spot activity: large non-axisymmetric spot structures, often on high latitudes and even forming polar caps (see e.g. Strassmeier 2009). This is true for evolved stars, e.g. RS CVn and FK Comae-type stars, and for young solar-type stars (see e.g. Willamo et al. 2019). These high-latitude structures have been confirmed, not only by Doppler imaging, but also using long-baseline infrared interferometry (Roettenbacher et al. 2016, 2017). The reason behind the similarities of these late-type stars in very different evolutionary stages is apparently a common kind of dynamo mechanism operating in their convection zone. The general notion is that rapid rotation suppresses the differential rotation, which could lead to dynamos of $\alpha^{2}$ - or $\alpha^{2} \Omega$ type, in contrast to the $\alpha \Omega$ dynamo operating in the Sun (see e.g. Ossendrijver 2003, and references therein).

There is no doubt that the photometric rotation period of HD 199178 is around 3.3 (see e.g. Jetsu et al. 1999). Furthermore, Jetsu et al. (1999) showed that the light curve of the star cannot be explained with a single, unique period. Jetsu et al. (1999) applied the Kuiper method on the times of the photometric minima and retrieved the period $P \approx 3.3175$ implying a long-lived active longitude. Panov \& Dimitrov (2007) used a mean period of $P \approx 3.30025$ to minimise the systematic drift 
of the phase of the photometric minimum. The variations in the photometric rotation period of HD 199178 were interpreted by Jetsu et al. (1999) as signs of differential rotation. In a recent study using long-term photometry of FK Comae, Jetsu (2018) suggested that the light curve is better explained with a combination of two periods. The interference between these two periods would cause apparent period variations, when fitting a single period model to the data. Thus estimating differential rotation from period variations in a model that is too simple may be misleading. However, two co-existing periods may itself be a consequence of differential rotation. As HD 199178 belongs to the same class as FK Comae, there are strong reasons to doubt that its differential rotation could be estimated by studying variations of a single period fit.

O’Neal et al. (1996, 1998) estimated starspot parameters of HD 199178, among other stars, using photometry and molecular bands. They reported the values $T_{\mathrm{Q}}=5350 \mathrm{~K}$ and $T_{\mathrm{S}}=$ $3800 \mathrm{~K}$ for the "quiet" surface and spots, respectively. Previous Doppler imaging studies of HD 199178 include the papers by Vogt (1988), Strassmeier et al. (1999), Hackman et al. (2001), and Petit et al. (2004). All of these revealed large polar or high-latitude spot structures. Petit et al. (2004) also calculated surface magnetic field maps for HD 199178 applying ZeemanDoppler imaging (ZDI) on Stokes $V$ spectropolarimetry obtained in 1998-2003. The common features in the ZDI maps presented in their study were mixed polarity radial fields ranging from -500 to $+500 \mathrm{G}$ in the polar region, and azimuthal fields forming rings or arches around the polar region.

The attempts to determine the differential rotation of HD 199178 using Doppler imaging have yielded conflicting results. Petit et al. (2004) employed differential rotation in the ZDI inversion and derived the value $\alpha \approx 0.041$ based on the goodness of the Doppler imaging solution. However, Hackman et al. (2001) tried different values of $\alpha$ and concluded that a negative $\alpha$ gave the best fit and smallest systematic error. As the general notion is that rapidly rotating single stars should not have strong anti-solar differential rotation, the latter result has been questioned (e.g. Rice 2002).

Several studies have aimed at determining the possible cyclic behaviour in the spot activity of HD 199178. Jetsu et al. (1990) reported a 9.07-year cycle in $U B V R I$-photometry, but failed to confirm it in a later study (Jetsu et al. 1999). Panov \& Dimitrov (2007) reported a cycle of 4.2 years in spot longitudes, while Savanov (2009) derived an eight-year brightness cycle.

In this study we present new Doppler images for HD 199178 using high-resolution spectroscopy collected with three different instruments in 1994-2017. Despite some gaps, this data set constitutes one of the longest series of Doppler images for any star. This unique series of Doppler images enables us to construct the equivalent of a solar butterfly diagram. Such diagrams are important for studying stellar dynamos, and have previously been constructed using three different approaches: by modelling light curves (see e.g. Livshits et al. 2003), based on Doppler imaging (see e.g. Hackman et al. 2011; Willamo et al. 2019), and utilising asteroseismology (Bazot et al. 2018; Nielsen et al. 2019).

\section{Observations}

The first observational set, July 1994, was collected with the $2 \mathrm{~m}$ Ritchey-Chretién telescope of the National Astronomical Observatory (Rozhen), Bulgaria. The rest of the data was obtained with the Nordic Optical Telescope (NOT) from August 1994 to December 2017. The NOT observations were obtained with the SOFIN spectrograph during 1994-2012 and in June 2017.
During 2013-2016 and in December 2017 the FIES spectrograph was used.

Seven spectral regions were used for Doppler imaging: 6263.0-6267.0 $\AA$ (strongest lines Fe I 6265.13 $\AA$ and V I $6266.31 \AA), 6409.6-6413.4 \AA$ (Fe I 6411.65 $\mathrm{A})$, 6428.5$6434.5 \AA$ (Fe I $6430.84 \AA$ and Fe II $6432.68 \AA$ ), $6437.0-6441.0 \AA$ (Ca I $6439.08 \AA$ ), 6641.6-6645.4 $\mathrm{A}$ (Ni I 6643.63 $\AA$ ), 6661.3$6665.3 \AA$ (Fe $\mathrm{I}$ 6663.23 $\AA$ and $\mathrm{Fe}_{\mathrm{I}}$ 6663.44 $\mathrm{A}$ ), and 7509.0$7513.4 \AA$ ( $F_{\text {I }} 7511.02 \AA$ ). A summary of all the observations is given in Table 1. As can be seen from the summary, different seasons covered different wavelength regions. However, the region with the line Ca I $6539.08 \AA$ was included in every data set. The signal-to-noise ratio was usually around 200-300. Spectra with $\mathrm{S} / \mathrm{N}$ as poor as $\sim 100$ were used in a few cases. The average $\mathrm{S} / \mathrm{N}$ is listed in Table 1.

The observations from July 1994 to July 1998 were already published (Hackman et al. 2001; Hackman 2004). They were reduced using the $3 \mathrm{~A}$ package (Ilyin 2000). The rest of the SOFIN data was reduced using a new reduction pipeline developed by the same author. A more detailed description of the new reduction pipeline can be found in Willamo et al. (2019) and will also be presented by Ilyin (in prep.). The FIES data was reduced using the FIEStool (Telting et al. 2014). The reduction covered all standard reduction procedures for high-resolution CCD spectra. The final continuum normalisation was done separately for each wavelength region (i.e. line blend) by comparing the observed spectrum to the synthetic spectrum during the Doppler imaging inversion (see Sect. 4). Plots of all spectral data together with the Doppler imaging modelled spectra, calculated using the adopted stellar parameters (Table 2), are shown in Appendix A.

In general, a minimum number of $\sim 10$ evenly distributed rotational phases is considered optimal for a fully reliable Doppler image. However, Hackman et al. (2011) demonstrated, that some useful information can be retrieved with a much more limited phase coverage. Therefore, we calculated Doppler images for all sets with $n_{\phi} \geq 4$.

\section{Spectral and stellar parameters}

The spectral parameters were obtained from the Vienna Atomic Line Database (Piskunov et al. 1995; Ryabchikova et al. 2015). Some adjustments were made in the $g f$-values in order to get a satisfactory fit for all spectral regions. This is a standard procedure in Doppler imaging since the spectra of real stars always show some deviations from calculations based on LTE-models. As we did not intend to derive exact element abundances, it is safe to correct for all discrepancies between the model and the real star by adjusting the $g f$-values. Furthermore, to the first order all photospheric absorption lines will follow the same behaviour (i.e. a cool spot will cause a "bump" in the spectral line).

The adopted parameters for the strongest lines are listed in Table 3. The largest adjustment was for the line Ca I $6439.075 \AA$, were VALD listed $\lg (g f)=0.370$ and we adopted the value 0.450 . A total number of 917 lines were included in the line synthesis of the seven wavelength regions. Most of these were molecular lines. For the unspotted surface a smaller number, i.e. only the atomic lines, would have been sufficient. For the cool spots molecular lines, in particular TiO-bands, are important. The local line profiles were calculated using the code LOCPRF7 developed at the University of Uppsala. Recent changes in the code include a molecular equilibrium solver (Piskunov \& Valenti 2017). 
Table 1. Summary of spectral observations.

\begin{tabular}{|c|c|c|c|c|c|c|c|c|}
\hline Month & $\langle\mathrm{HJD}\rangle-2400000$ & $R$ & $n_{\phi}$ & $\Delta t(\mathrm{~d})$ & $f_{\phi}$ & Wavelength regions $(\AA)$ & $\langle S / N\rangle$ & $\sigma(\%)$ \\
\hline July 1994 & 49558.5 & 35000 & 19 & 14 & $98 \%$ & 6431,6439 & 239 & 0.529 \\
\hline August 1994 & 49583.5 & 80000 & 11 & 10 & $90 \%$ & $6411,6431,6439,7511$ & 265 & 0.455 \\
\hline November 1994 & 49673.8 & 140000 & 9 & 9 & $86 \%$ & 6431,6439 & 206 & 0.540 \\
\hline July 1995 & 49916.5 & 80000 & 11 & 11 & $95 \%$ & $6411,6431,6439,7511$ & 333 & 0.381 \\
\hline October 1996 & 50384.8 & 80000 & 8 & 7 & $75 \%$ & $6411,6431,6439,7511$ & 326 & 0.408 \\
\hline June 1997 & 50623.0 & 80000 & 12 & 10 & $97 \%$ & $6411,6431,6439,7511$ & 278 & 0.435 \\
\hline July 1998 & 51003.8 & 80000 & 13 & 13 & $96 \%$ & $6411,6431,6439,7511$ & 320 & 0.480 \\
\hline October 1998 & 51092.1 & 80000 & 10 & 8 & $85 \%$ & $6411,6431,6439,7511$ & 244 & 0.546 \\
\hline November 1998 & 51124.4 & 80000 & 7 & 6 & $69 \%$ & $6411,6431,6439,7511$ & 230 & 0.520 \\
\hline May 1999 & 51328.2 & 80000 & 14 & 10 & $93 \%$ & $6411,6431,6439,7511$ & 203 & 0.617 \\
\hline July 1999 & 51388.5 & 80000 & 11 & 10 & $97 \%$ & $6411,6431,6439,7511$ & 174 & 0.694 \\
\hline October 1999 & 51474.0 & 80000 & 9 & 9 & $64 \%$ & $6411,6431,6439,7511$ & 192 & 0.780 \\
\hline August 2000 & 51768.6 & 80000 & 21 & 11 & $100 \%$ & $6431,6439,7511$ & 222 & 0.618 \\
\hline June 2001 & 52068.4 & 80000 & 15 & 7 & $89 \%$ & $6431,6439,7511$ & 201 & 0.502 \\
\hline August 2002 & 52511.8 & 80000 & 23 & 9 & $100 \%$ & $6411,6431,6439$ & 259 & 0.524 \\
\hline November 2002 & 52594.3 & 80000 & 8 & 12 & $68 \%$ & $6411,6431,6439$ & 285 & 0.399 \\
\hline June 2003 & 52803.4 & 80000 & 16 & 19 & $95 \%$ & $6411,6431,6439$ & 268 & 0.462 \\
\hline November 2003 & 52950.6 & 80000 & 18 & 20 & $100 \%$ & $6411,6431,6439$ & 254 & 0.515 \\
\hline August 2004 & 53220.2 & 80000 & 16 & 13 & $89 \%$ & $6411,6431,6439$ & 257 & 0.527 \\
\hline July 2005 & 53571.4 & 80000 & 11 & 16 & $75 \%$ & $6411,6431,6439$ & 270 & 0.500 \\
\hline November 2005 & 53690.8 & 80000 & 6 & 10 & $50 \%$ & $6411,6431,6439$ & 242 & 0.525 \\
\hline September 2006 & 53982.6 & 80000 & 13 & 9 & $91 \%$ & $6411,6431,6439$ & 280 & 0.514 \\
\hline December 2006 & 54074.8 & 80000 & 8 & 7 & $75 \%$ & $6265,6439,6644$ & 211 & 0.728 \\
\hline July 2007 & 54305.9 & 80000 & 12 & 10 & $94 \%$ & $6411,6431,6439$ & 238 & 0.540 \\
\hline September 2008 & 54721.2 & 80000 & 7 & 6 & $60 \%$ & $6265,6431,6439,6644$ & 337 & 0.494 \\
\hline December 2008 & 54811.8 & 80000 & 6 & 6 & $49 \%$ & $6265,6431,6439,6644$ & 213 & 0.835 \\
\hline August 2009 & 55074.7 & 80000 & 9 & 11 & $78 \%$ & $6439,6644,6663$ & 297 & 0.663 \\
\hline September 2009 & 55075.7 & 80000 & 11 & 12 & $80 \%$ & $6411,6439,6644$ & 259 & 0.524 \\
\hline December 2009 & 55197.1 & 80000 & 5 & 6 & $47 \%$ & $6265,6439,6644$ & 205 & 0.536 \\
\hline July 2010 & 55402.2 & 80000 & 10 & 13 & $89 \%$ & $6265,6439,6644$ & 287 & 0.551 \\
\hline December 2010 & 55552.6 & 80000 & 4 & 12 & $31 \%$ & $6265,6439,6644$ & 266 & 0.422 \\
\hline December 2011 & 55908.8 & 80000 & 4 & 4 & $40 \%$ & $6265,6439,6644$ & 355 & 0.469 \\
\hline August 2012 & 56170.5 & 80000 & 9 & 7 & $87 \%$ & $6265,6439,6644$ & 349 & 0.382 \\
\hline November 2012 & 56259.6 & 80000 & 4 & 11 & $40 \%$ & $6265,6439,6644$ & 232 & 0.540 \\
\hline August 2014 & 56886.0 & 67000 & 10 & 9 & $84 \%$ & $6265,6411,6431,6439,6644$ & 352 & 0.382 \\
\hline December 2014 & 56996.2 & 67000 & 6 & 6 & $58 \%$ & $6265,6411,6431,6439,6644$ & 293 & 0.431 \\
\hline July 2015 & 57209.1 & 67000 & 9 & 9 & $78 \%$ & $6265,6411,6431,6439,6644$ & 380 & 0.380 \\
\hline November 2015 & 57355.9 & 67000 & 7 & 8 & $69 \%$ & $6265,6411,6431,6439,6644$ & 246 & 0.481 \\
\hline June 2016 & 57560.0 & 67000 & 8 & 8 & $75 \%$ & $6265,6411,6431,6439,6644$ & 318 & 0.663 \\
\hline June 2017 & 57914.4 & 80000 & 7 & 10 & $59 \%$ & $6265,6431,6439,6644$ & 240 & 0.451 \\
\hline December 2017 & 58108.3 & 67000 & 5 & 4 & $49 \%$ & $6265,6411,6431,6439,6644$ & 245 & 0.636 \\
\hline
\end{tabular}

Notes. $\langle\mathrm{HJD}\rangle$ is the mean heliocentric Julian date, $R$ the spectral resolution, $n_{\phi}$ the number of observations, $\Delta t$ the time span of the observations, $f_{\phi}$ the phase coverage of the season (see Sect. 4 for a definition), $\langle S / N\rangle$ the mean signal-to-noise ratio, and $\sigma$ the mean deviation between the observations and DI solution.

HD 199178 is a rapidly rotating late-type star, which was classified as G 5 III - IV by Herbig (1958). Strassmeier et al. (1999) calculated its luminosity and mass to be $11 L_{\odot}$ and $1.65 M_{\odot}$. Hackman et al. (2001) estimated its radius to be $\sim 5 R_{\odot}$ and adopted the rotation velocity $v \sin i=72 \mathrm{~km} \mathrm{~s}^{-1}$, inclination angle $i=60^{\circ}$, and gravity $\lg (g)=3.5$. With such a high rotation velocity, the macroturbulence is hard to determine, but in any case should not strongly influence Doppler imaging. In this study we used a radial-tangential macroturbulence of $\zeta_{\mathrm{RT}}=5.0 \mathrm{~km} \mathrm{~s}^{-1}$. We adopted an effective temperature $T_{\text {eff }}=5300 \mathrm{~K}$ for the unspotted surface as a starting point. The microturbulence was determined by comparing the synthetic spectrum of an unspotted surface to the observations. This yielded $\xi=1.7 \mathrm{~km} \mathrm{~s}^{-1}$.
Jetsu et al. (1999) and Panov \& Dimitrov (2007) concluded, that the photometric light curve cannot be modelled using a constant rotation period. However, in order to compare temperature maps of different seasons, all observations have to be phased by a single rotation period. This is particularly important because of our intention to study possible active longitudes and/or azimuthal dynamo waves. Thus, we adopted the period $P_{\mathrm{al}}=3.3175$, which was reported by Jetsu et al. (1999) as the most significant one in the analysis of possible long-term active longitudes. Even though this may not be fully correct for all seasons, each observation run is short enough that a small error in the period will not distort the image. The phases for the observations were obtained with the 
Table 2. Adopted stellar parameters for HD 199178.

\begin{tabular}{lcl}
\hline \hline Parameter & Adopted & Reference $^{(a)}$ \\
\hline Gravity $\lg (g)$ & 3.5 & Hackman et al. (2001) \\
Inclination $i$ & $60^{\circ}$ & Hackman et al. (2001) \\
Rotation velocity & $72 \mathrm{~km} \mathrm{~s}^{-1}$ & Hackman et al. (2001) \\
Rotation period & 3.3175 & Jetsu et al. (1999) \\
Differential rotation $\alpha$ & 0 & \\
Micro turbulence $\xi$ & $1.7 \mathrm{~km} \mathrm{~s}^{-1}$ & \\
Macro turbulence $\zeta_{\mathrm{RT}}$ & $5.0 \mathrm{~km} \mathrm{~s}^{-1}$ & \\
Element abundances & Solar & Strassmeier et al. (1999) \\
\hline
\end{tabular}

Notes. ${ }^{(a)}$ In case of no reference, a new parameter value was derived/adopted for this study.

Table 3. Wavelengths, lower excitation potentials, and adopted $\lg (g f)$ values for the strongest lines.

\begin{tabular}{lcrr}
\hline \hline Line & $\lambda(\AA)$ & $\chi_{\text {low }}(\mathrm{eV})$ & $\lg (g f)$ \\
\hline $\mathrm{Fe}_{\mathrm{I}}$ & 6265.1319 & 2.1759 & -2.550 \\
$\mathrm{~V}_{\mathrm{I}}$ & 6266.3069 & 0.2753 & -2.290 \\
$\mathrm{Fe}_{\mathrm{I}}$ & 6411.6476 & 3.6537 & -0.705 \\
$\mathrm{Fe}_{\mathrm{I}}$ & 6430.8446 & 2.1759 & -2.206 \\
$\mathrm{Fe}_{\mathrm{II}}$ & 6432.6757 & 2.8910 & -3.320 \\
$\mathrm{Ca}_{\mathrm{I}}$ & 6439.0750 & 2.5257 & 0.450 \\
$\mathrm{Ni}_{\mathrm{I}}$ & 6643.6304 & 1.6764 & -1.920 \\
$\mathrm{Fe}_{\mathrm{I}}$ & 6663.2311 & 4.5585 & -1.239 \\
$\mathrm{Fe}_{\mathrm{I}}$ & 6663.4407 & 2.4242 & -2.479 \\
$\mathrm{Fe}_{\mathrm{I}}$ & 7511.0181 & 4.1777 & 0.199 \\
\hline
\end{tabular}

ephemeris

$\mathrm{HJD}_{\phi=0}=2449557.664+3.3175 \times E$.

The surface differential rotation parameter $\alpha$ is usually expressed as

$\alpha=\frac{\Omega_{\mathrm{eq}}-\Omega_{\mathrm{pole}}}{\Omega_{\mathrm{eq}}}$,

where $\Omega_{\mathrm{eq}}$ and $\Omega_{\text {pole }}$ are the angular rotation velocities at the equator and approaching the pole, respectively. In the Sun the differential rotation follows the approximate profile

$\Omega(\theta) \approx \Omega_{\mathrm{eq}}\left(1-\alpha \sin ^{2} \theta\right)$.

Here $\theta$ is the stellar latitude. It should be noted that Eq. (3) has only been proven valid for the Sun. Numerical simulations show that faster rotating stars could have completely different differential rotation curves (see e.g. Kitchatinov \& Rüdiger 1999, 2004). Still the solar differential rotation curve is, for lack of a better option, used as a model for other stars.

In the study by Hackman et al. (2001) the best fit for the Doppler images was achieved using the combination $v \sin i=$ $70 \mathrm{~km} \mathrm{~s}^{-1}, \alpha=-0.17$ and $P_{\text {eq }}=3.81$. For the present study, we tested the cases of $\alpha=0, \alpha=-0.17$, and $\alpha=0.034$, the last value corresponding to the differential rotation derived for the epoch 2001.97 by Petit et al. (2004). The reason for choosing this value instead of $\alpha=0.041$ is that the latter was a weighted mean and each value of $\alpha$ had a specific corresponding $\Omega_{\mathrm{eq}^{-}}$ value. Using weighted means for both of these parameters would not necessarily have formed a consistent pair. In these tests we
Table 4. Convergence of DI solutions for different values of $\alpha$ in TEST1 and TEST2.

\begin{tabular}{lclcccc}
\hline \hline & $\alpha$ & $P_{\mathrm{eq}}$ & $v \sin i$ & $\sigma_{0}$ & $\sigma_{20}$ & $\sigma_{150}$ \\
\hline \multirow{2}{*}{ TEST1 } & -0.17 & 3.81 & $70 \mathrm{~km} \mathrm{~s}^{-1}$ & $0.645 \%$ & $0.539 \%$ & $0.502 \%$ \\
& 0.0 & 3.3175 & $72 \mathrm{~km} \mathrm{~s}^{-1}$ & $0.737 \%$ & $0.564 \%$ & $0.515 \%$ \\
& 0.034 & 3.2488 & $73 \mathrm{~km} \mathrm{~s}^{-1}$ & $0.721 \%$ & $0.567 \%$ & $0.508 \%$ \\
\hline TEST2 & -0.17 & 3.81 & $70 \mathrm{~km} \mathrm{~s}^{-1}$ & $0.619 \%$ & $0.548 \%$ & $0.531 \%$ \\
& 0.0 & 3.3175 & $72 \mathrm{~km} \mathrm{~s}^{-1}$ & $0.701 \%$ & $0.596 \%$ & $0.544 \%$ \\
& 0.034 & 3.2488 & $73 \mathrm{~km} \mathrm{~s}^{-1}$ & $0.710 \%$ & $0.599 \%$ & $0.547 \%$ \\
\hline
\end{tabular}
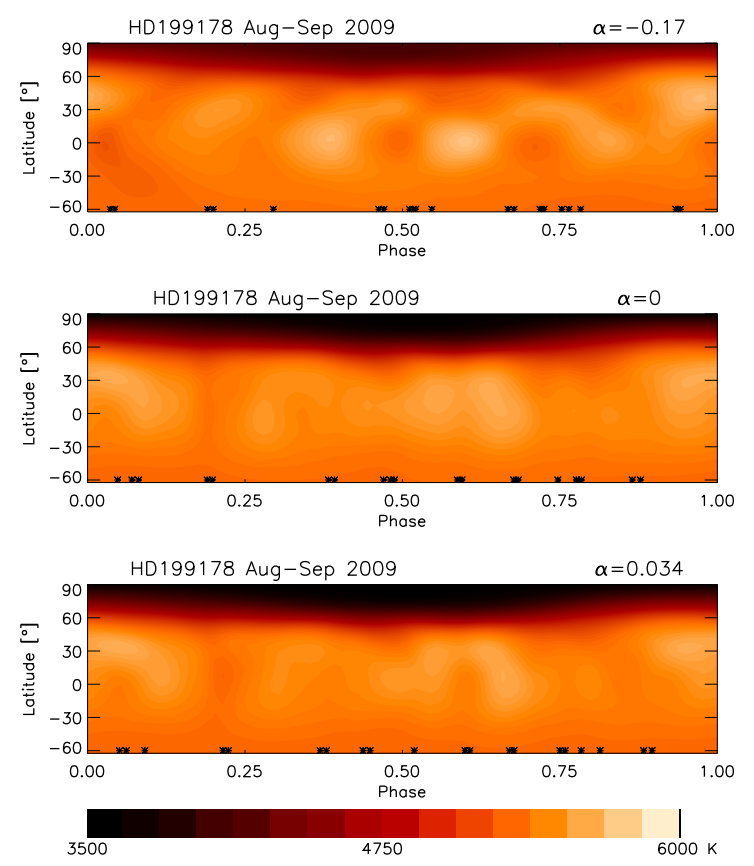

Fig. 1. DI surface temperature maps of TEST2. The observed phases are indicated with "**" and the same temperature scale is used in the three images.

accounted for both the surface shearing effect and change in the rotational profile caused by differential rotation. For the test we chose two sets. We can assume that any differential rotation will be detected most easily when the observation set covers a long period and the phase coverage is dense. Thus, we chose the longest set, November 2003 (TEST1), and combined the August and September 2009 sets into one set with better phase coverage (TEST2). In the latter case we could only use the common wavelength regions $6439 \AA$ and $6644 \AA$. Before the tests we chose the optimal $v \sin i$ for each $\alpha$ value by testing values with a grid of $1 \mathrm{~km} \mathrm{~s}^{-1}$. The resulting deviation between the observations and the Doppler imaging (DI) solution after 0, 20, and 150 iterations $\left(\sigma_{0}, \sigma_{20}\right.$, and $\left.\sigma_{150}\right)$ are shown in Table 4 . When running the inversions for a sufficient number of iterations (150 iterations) the differences in the deviation of the fits were small. The DI temperature maps for the August-September 2009 set are shown in Fig. 1.

We thus confirmed the previous result by Hackman et al. (2001) that using an anti-solar differential rotation will lead to a faster convergence and slightly smaller deviation of the Doppler imaging solution. An anti-solar differential rotation as strong as $\alpha=-0.17$ would be surprising taking into account theoretical and observational results. Firstly, only slowly rotating stars should have anti-solar differential rotation (Gastine et al. 2014), although there have been studies reporting weak anti-solar 
differential rotation for some RS CVn binary components (see e.g Harutyunyan et al. 2016). Secondly, rapidly rotating stars should not have such a strong differential rotation (see e.g. Küker \& Rüdiger 2011; Reinhold \& Gizon 2015). However, HD 199178 differs significantly from most rapidly rotating latetype stars in that it has likely undergone a merger in the transit from a W Uma-type binary to a FK Comae-type star (see e.g. Bopp \& Rucinski 1981). Furthermore, asteroseismologic studies report strong internal shears as a result of core contraction in stars of similar luminosity class (e.g. Deheuvels et al. 2012). It is unclear what imprints a merger and possibly a strong radial shear would leave at the surface.

Nevertheless, we believe that the approach of fastest convergence and/or smallest deviation is not the correct way to determine stellar rotational parameters like $\alpha$. One reason for this is the regularisation function (e.g. maximum entropy or minimum local gradient), which will in practice favour solutions with less deviation from a constant surface. By manipulating stellar rotation parameters, axisymmetric spot structures can be minimised and the inversion procedure will thus favour this set of parameters. As has been demonstrated in several studies (see e.g. Hackman et al. 2001), an anti-solar differential rotation will have an effect on rotationally broadened spectral line profiles similar to the effect of a large polar spot. Such high-latitude structures have been confirmed independently using interferometric observations (Roettenbacher et al. 2016).

The conclusion is that the rotational parameters cannot be determined merely by optimising the Doppler imaging solution. The best way to determine the differential rotation would be to trace the movement of spot structures versus their latitude. The problem with this approach in our case is that the reliable cool spot structures in HD 199178 are found in a limited latitude range near the rotation pole (see e.g. Petit et al. 2004, and Fig. 2 in the present study).

In conclusion: The differential rotation of HD 199178 is expected to be small and the determination of it is not possible with the present spectroscopic data. Thus, we chose to adopt the value $\alpha=0$ as this allows for unambiguous rotation phases for the whole set of images.

\section{Doppler imaging}

We used the Doppler imaging code INVERS7DR originally written at the University of Uppsala, with some changes made at the University of Helsinki (see e.g. Piskunov 1991; Hackman et al. 2012). A total of 41 new images were calculated for both the previously used spectra from 1994 to 1998 and all new spectra. A table of synthetic spectra was first calculated for a set of temperatures $(3500-6000 \mathrm{~K})$ and a number of limb angles $\left(n_{\mu}=40\right)$ using stellar model atmospheres supplied by MARCS (Gustafsson et al. 2008). This table was then used to solve the inverse problem, i.e. searching for the surface temperature distribution that best reproduces the observed photospheric spectra. In order to regularise this ill-posed inversion problem, Tikhonov regularisation was employed. This means adding the additional constraint of a minimum surface gradient of the solution. Furthermore, the solution was limited to the temperature range of the used model atmospheres (i.e. $3500-6000 \mathrm{~K}$ ) by an additional penalty function similar to the one described by Hackman et al. (2001).

In general, the method of regularisation is of less importance than the calculation of the synthetic line profiles. However, with a high inclination angle $\left(\geq 60^{\circ}\right)$ the region at the rotational pole will always be near the stellar limb and have a limited effect on the observed line profiles. Therefore, the Tikhonov regularisation may cause the convergence of the solution to proceed slowly just at the pole. Thus, a large number of iterations are needed to map this region correctly. In this study we used 150 iterations and a regularisation parameter of $\Lambda=1 \times 10^{-9}$. All DI surface temperature maps are displayed in equirectangular projection in Fig. 2. The spectra calculated from the DI solutions are displayed in Appendix A.

Based on the assumption that ten evenly distributed rotational phases would cover the whole star, we estimated a phase coverage $f_{\phi}$ for each DI (listed in Table 1). The mean phase coverage was $\left\langle f_{\phi}\right\rangle \approx 77 \%$. In six cases (December 2008, December 2009, December 2010, December 2011, November 2012, and December 2017) $f_{\phi}$ was less than $50 \%$.

The August 2009 and September 2009 observations are actually simultaneous; there is a difference in $\langle\mathrm{HJD}\rangle$ of only one day. However, the images were derived using different spectral setups. This was a robust test for differences in the resulting DI caused by using different spectral lines and phase coverage. The polar spots in these images are almost identical, except for slight differences around phases near $\phi=0$. This difference can be explained by more observations near this phase in the case of September 2009. Otherwise, the only visible differences in these images are the weak lower latitude features, which are thus most probably artefacts.

\section{Results}

We see high-latitude spot structures in all DIs (Fig. 2). If maps based on observations with $f_{\phi}<50 \%$ are regarded as unreliable, we can also conclude that all reliable images show a large polar spot structure. This structure is varying in time, and the rotationally modulated signal is dominated by annexes extending towards lower latitudes. This polar spot structure is usually limited to latitudes $\theta>60^{\circ}$; sometimes the annex extends to $\theta \approx 40^{\circ}$. There are also lower latitude weaker features, particularly during November 1998, September 2008, November 2015, and June 2017, which could contribute to the light curve modulation. However, these cases are all images with $f_{\phi}<70 \%$ and the lower latitude cool spot structures have nearby hot structures, indicating that they may be artefacts (see Hackman et al. 2001).

Images near in time allow us to study short-term changes in the spot configuration. In 1994 (July and August) and 1998 (October and November) we have images separated by about one month. In both cases we see some small differences, but the overall spot configuration remains unchanged. Images with a longer time difference (e.g. May and July 1999) already show significant differences that cannot be explained merely by phase shifts caused by rotation period uncertainties.

To study the long-term spot evolution we present the temporal variation in the longitudinally and latitudinally averaged temperatures (Figs. 3 and 4). The former is an analogue to the butterfly diagram, while the latter can be used to detect stable or drifting active longitudes. These structures have been revealed in observations (see e.g. Lindborg et al. 2011; Hackman et al. 2011) and in numerical simulations (Cole et al. 2014; Viviani et al. 2018).

In Fig. 4 the phases correspond to the frame rotating according to the ephemeris of Eq. (1). Figure 3 indicates a smooth behaviour, when disregarding the most unreliable images based on insufficient phase coverage $\left(f_{\phi}<50 \%\right)$. Figure 4 gives no indication of active longitudes.

We also calculated the mean surface temperatures $T_{\text {mean }}$ and spot filling factors $f_{\text {spot }}$ for each DI. We used $T_{\text {spot }}=4800 \mathrm{~K}$ 

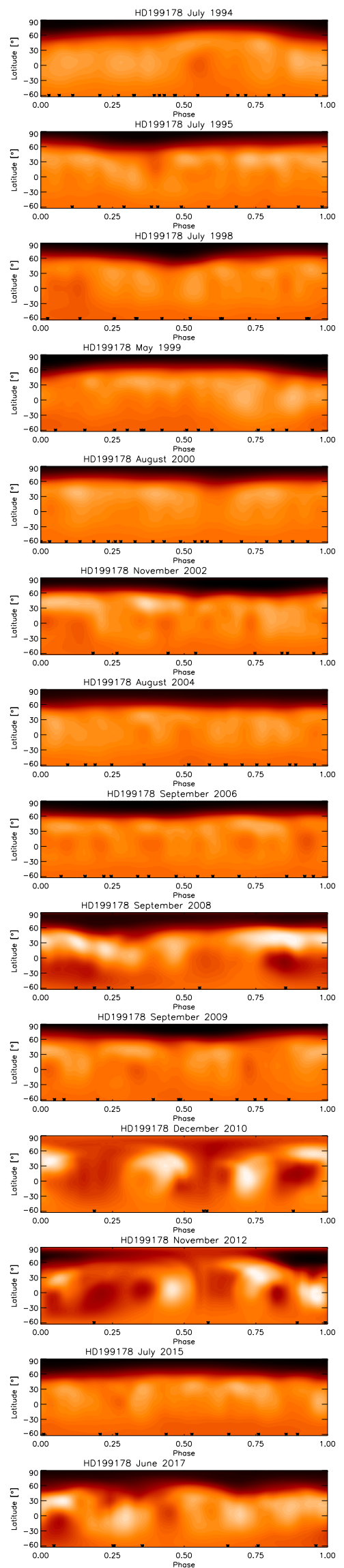
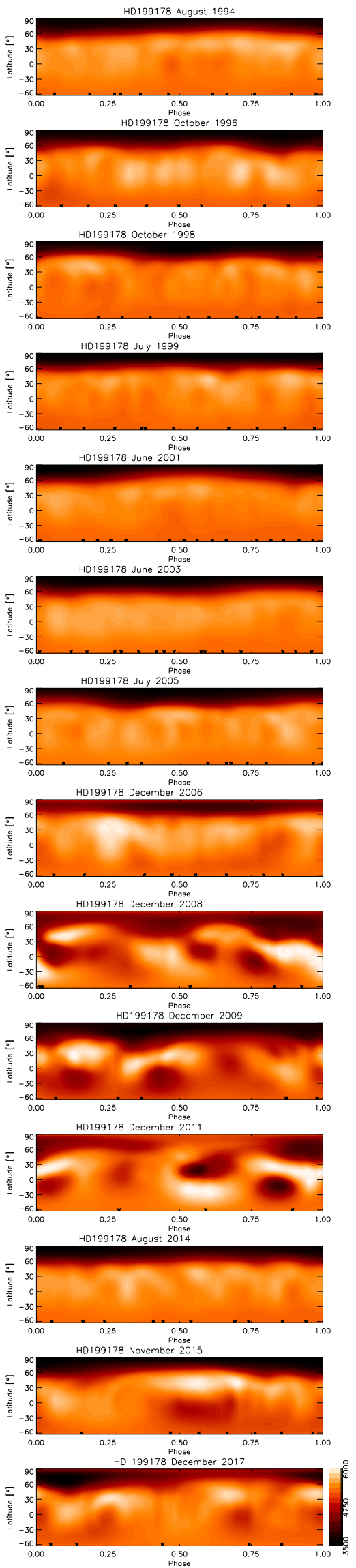
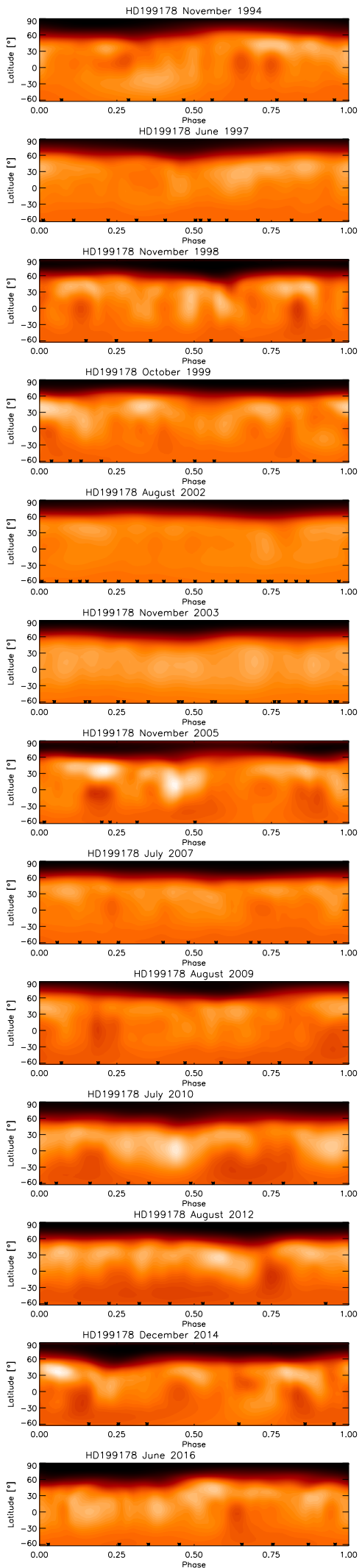

Fig. 2. Doppler imaging surface temperature maps. The observed phases are indicated with "**" and the same temperature scale is used in all images. 


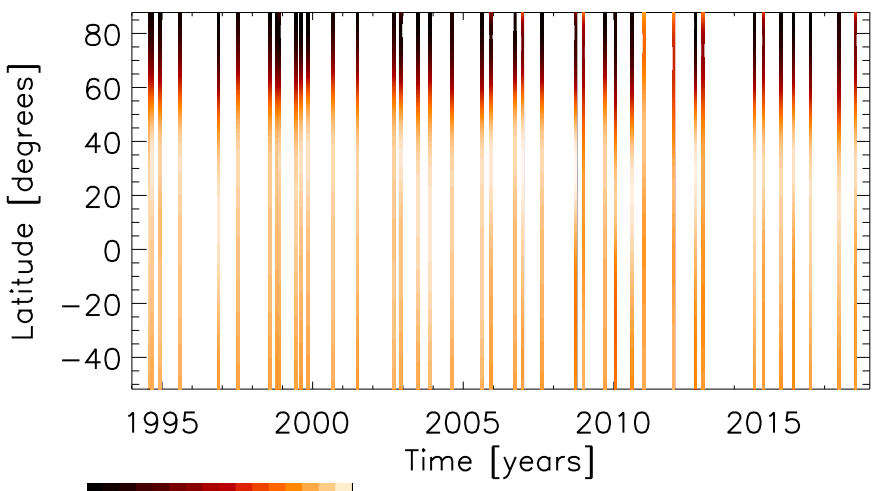

$35034559 \quad 5616 \mathrm{~K}$

Fig. 3. DIs averaged over longitudes vs. dates: time-latitude diagram.

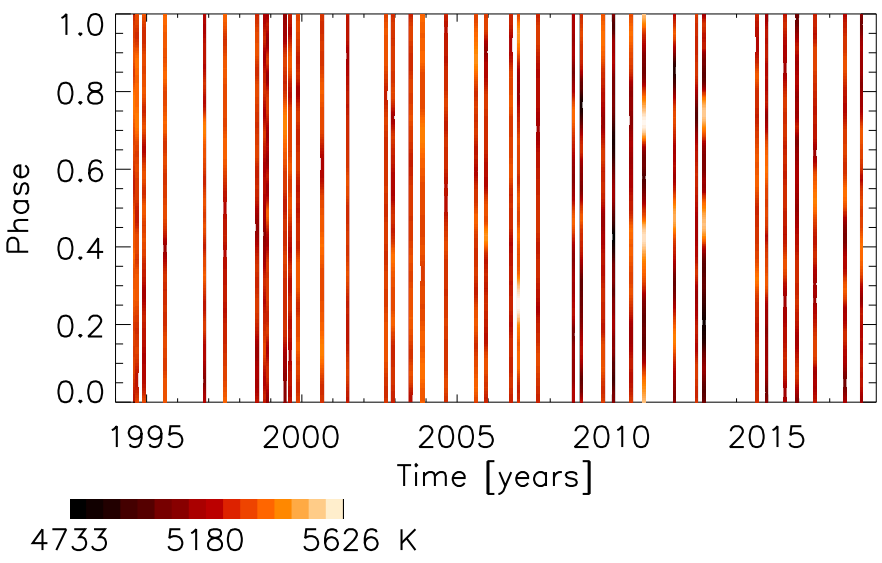

Fig. 4. DIs averaged over latitudes vs. dates: time-longitude diagram.

as the spot threshold. This is $500 \mathrm{~K}$ lower than the assumed unspotted surface $T_{\text {eff }}=5300 \mathrm{~K}$. The results are plotted in Figs. 5 and 6. The linear Pearson's correlation between $T_{\text {mean }}$ and $f_{\text {spot }}$ of the reliable maps $\left(f_{\phi}>0.5\right)$ was $r\left(T_{\text {mean }}, f_{\text {spot }}\right) \approx$ -0.60 . It should be pointed out that the chosen threshold is, in a sense, arbitrary, but the correlation did not change significantly when using slightly different values. For example, using $T_{\text {spot }}=5000 \mathrm{~K}$ gave $r\left(T_{\text {mean }}, f_{\text {spot }}\right) \approx-0.60$ and $T_{\text {spot }}=4500 \mathrm{~K}$ yielded $r\left(T_{\text {mean }}, f_{\text {spot }}\right) \approx-0.55$.

\section{Conclusions}

We have presented an extremely long series of 41 DI surface temperature maps of HD 199178, including all but one year during 1994-2017. The dominating spot structure is a large spot/spot group covering the region around the rotational pole and extending down to latitudes $40^{\circ}-70^{\circ}$ depending on the season. This polar structure is clearly a stable phenomenon. It is usually off-centred from the rotational pole, thus causing the rotationally modulated variations in the observed line profiles of photospheric absorption lines. Some of our images show spot activity at lower latitudes, but in all cases this can be caused by artefacts due to limited phase coverage.

Some of our DIs are nearly simultaneous with the ZDIs presented by Petit et al. (2004). Assuming their surface magnetic radial fields are correct, then any magnetic origin of the large cool polar structure must come from smaller spot structures with opposite magnetic polarities. In this case nearby opposite polarities would cancel out in a ZDI, leading to difficulties

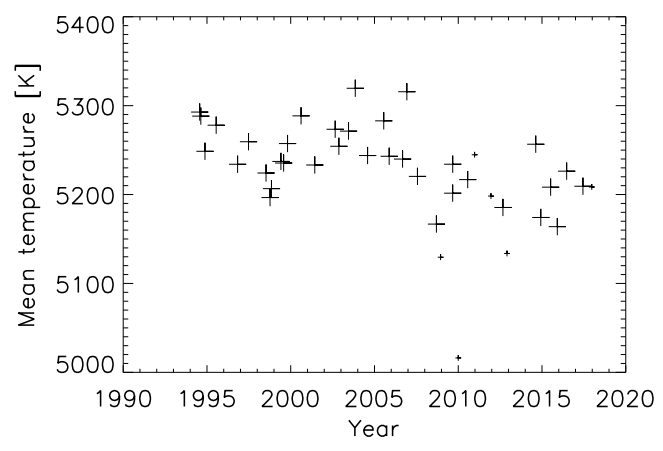

Fig. 5. Mean surface temperature $T_{\text {mean }}$ of the DI maps. Reliable maps $\left(f_{\phi} \geq 0.5\right)$ are represented with larger symbols than the unreliable ones.

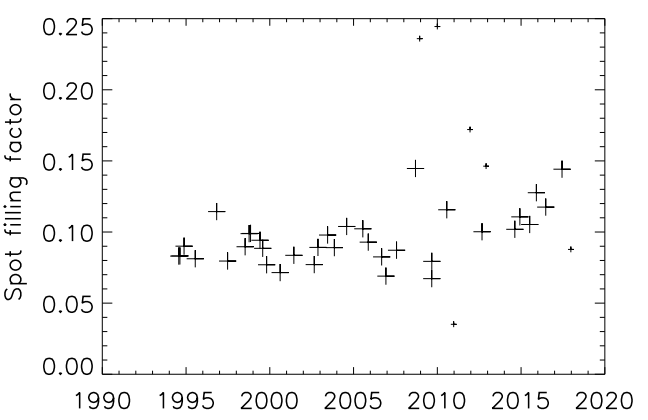

Fig. 6. Spot filling factors. Reliable maps $\left(f_{\phi} \geq 0.5\right)$ are represented with larger symbols than the unreliable ones.

in characterising the magnetic field associated with the largest cool spots. Another problem connected to correlating surface dark spots with magnetic fields is that the polarisation signal is weighted by the flux. Rosén \& Kochukhov (2012) showed that this may lead to an underestimation of the magnetic field in a cool spot.

Comparing images taken in the same years indicates that the spot configuration is stable for about a month, but significant differences can occur after two months. This is in line with the time scales of significant light curve changes for FK Comae (Hackman et al. 2013). By plotting the averages of the DIs over longitudes and latitudes (Figs. 3 and 4) we studied the long-term evolution of the spot configuration. The latitudinal spot distribution behaves smoothly while the longitudinal distribution shows no indication of active longitudes. This pattern differs somewhat from those derived for three other single rapidly rotating stars: FK Com (Hackman et al. 2013), LQ Hya (Cole et al. 2015), and V899 Her (Willamo et al. 2019). In these cases there were coherent structures visible for a few years, while HD 199178 seems more erratic in this sense.

The negative correlation between the mean temperature $T_{\text {mean }}$ and spot filling factor $f_{\text {spot }}$ shows that the variability is dominated by cool spots. The variations in $T_{\text {mean }}$ and $f_{\text {spot }}$ are not regular enough to draw conclusions on any activity cycles. There is, however, a tendency of a decrease in $T_{\text {mean }}$ since 2007 as well as a slight increase in $f_{\text {spot }}$ since 2010 .

By comparing two images derived from almost simultaneous data (August and September 2009) we demonstrate that the use of different wavelength regions will not cause any significant systematic differences in the resulting DI maps. This comparison also shows that the weaker contrast lower latitude structures may be artefacts.

Despite previously reported differential rotation (see e.g. Petit et al. 2004), we calculated our DI maps without differential 
rotation. Since the DIs do not show any reliable lower latitude spots, it is hard to even roughly estimate the differential rotation of the star. The adopted differential rotation parameter $\alpha=0$ also produced good fits for all seasons, and tests with other values did not significantly improve the goodness of the fit.

Concerning the derivation of rotational parameters, we demonstrate that the generally used approach of searching for the fastest or best convergence of the DI solution may be misleading. In particular, the possible differential rotation of HD 199178 cannot be determined with this approach.

Acknowledgements. This work has made use of the VALD database which is operated at Uppsala University, the Institute of Astronomy RAS in Moscow, and the University of Vienna. Part of the observations were obtained at the National Astronomical Observatory, Rozhen, Bulgaria. J.J.L. and M.J.K. were partially supported by the Academy of Finland Centre of Excellence ReSoLVE (project 307411). O.K. acknowledges support from the Knut and Alice Wallenberg Foundation, the Swedish Research Council, and the Swedish National Space Board. The work of T.W. was financed by the Emil Aaltonen Foundation. We thank the referee Dr. Pascal Petit for the valuable and constructive suggestions on how to improve the paper.

\section{References}

Bazot, M., Nielsen, M. B., Mary, D., et al. 2018, A\&A, 619, L9

Bopp, B. W., \& Rucinski, S. M. 1981, in Fundamental Problems in the Theory of Stellar Evolution, eds. D. Sugimoto, D. Q. Lamb, \& D. N. Schramm, IAU Symp., 93, 177

Cole, E., Käpylä, P. J., Mantere, M. J., \& Brandenburg, A. 2014, ApJ, 780, L22

Cole, E. M., Hackman, T., Käpylä, M. J., et al. 2015, A\&A, 581, A69

Deheuvels, S., García, R. A., Chaplin, W. J., et al. 2012, ApJ, 756, 19

Gastine, T., Yadav, R. K., Morin, J., Reiners, A., \& Wicht, J. 2014, MNRAS, 438, L76

Gustafsson, B., Edvardsson, B., Eriksson, K., et al. 2008, A\&A, 486, 951

Hackman, T. 2004, PhD Thesis, Observatory Report 1/2004, University of Helsinki, Finland

Hackman, T., Jetsu, L., \& Tuominen, I. 2001, A\&A, 374, 171

Hackman, T., Mantere, M. J., Jetsu, L., et al. 2011, Astron. Nachr., 332, 859

Hackman, T., Mantere, M. J., Lindborg, M., et al. 2012, A\&A, 538, A126
Hackman, T., Pelt, J., Mantere, M. J., et al. 2013, A\&A, 553, A40

Harutyunyan, G., Strassmeier, K. G., Künstler, A., Carroll, T. A., \& Weber, M. 2016, A\&A, 592, A117

Herbig, G. H. 1958, ApJ, 128, 259

Ilyin, I. V. 2000, PhD Thesis, Department of Physical Sciences, University of Oulu Finland

Jetsu, L. 2018, MNRAS, submitted [arXiv:1808.02221]

Jetsu, L., Huovelin, J., Tuominen, I., et al. 1990, A\&A, 236, 423

Jetsu, L., Pelt, J., \& Tuominen, I. 1999, A\&A, 351, 212

Kitchatinov, L. L., \& Rüdiger, G. 1999, A\&A, 344, 911

Kitchatinov, L. L., \& Rüdiger, G. 2004, Astron. Nachr., 325, 496

Küker, M., \& Rüdiger, G. 2011, Astron. Nachr., 332, 933

Lindborg, M., Korpi, M. J., Hackman, T., et al. 2011, A\&A, 526, A44

Livshits, M. A., Alekseev, I. Y., \& Katsova, M. M. 2003, Astron. Rep., 47, 562

Nielsen, M. B., Gizon, L., Cameron, R. H., \& Miesch, M. 2019, A\&A, 622, A85

O’Neal, D., Saar, S. H., \& Neff, J. E. 1996, ApJ, 463, 766

O’Neal, D., Neff, J. E., \& Saar, S. H. 1998, ApJ, 507, 919

Ossendrijver, M. 2003, A\&ARv, 11, 287

Panov, K., \& Dimitrov, D. 2007, A\&A, 467, 229

Petit, P., Donati, J.-F., Oliveira, J. M., et al. 2004, MNRAS, 351, 826

Piskunov, N. E. 1991, in IAU Colloq. 130: The Sun and Cool Stars. Activity, Magnetism, Dynamos, eds. I. Tuominen, D. Moss, \& G. Rüdiger (Berlin: Springer Verlag), Lect. Notes Phys., 380, 309

Piskunov, N., \& Valenti, J. A. 2017, A\&A, 597, A16

Piskunov, N. E., Kupka, F., Ryabchikova, T. A., Weiss, W. W., \& Jeffery, C. S. 1995, A\&AS, 112, 525

Reinhold, T., \& Gizon, L. 2015, A\&A, 583, A65

Rice, J. B. 2002, Astron. Nachr., 323, 220

Roettenbacher, R. M., Monnier, J. D., Korhonen, H., et al. 2016, Nature, 533, 217

Roettenbacher, R. M., Monnier, J. D., Korhonen, H., et al. 2017, ApJ, 849, 120

Rosén, L., \& Kochukhov, O. 2012, A\&A, 548, A8

Ryabchikova, T., Piskunov, N., Kurucz, R. L., et al. 2015, Phys. Scr., 90, 054005

Savanov, I. S. 2009, Astron. Rep., 53, 1032

Strassmeier, K. G. 2009, A\&ARv, 17, 251

Strassmeier, K. G., Lupinek, S., Dempsey, R. C., \& Rice, J. B. 1999, A\&A, 347, 212

Telting, J. H., Avila, G., Buchhave, L., et al. 2014, Astron. Nachr., 335, 41 Viviani, M., Warnecke, J., Käpylä, M. J., et al. 2018, A\&A, 616, A160

Vogt, S. S. 1988, in The Impact of Very High S/N Spectroscopy on Stellar Physics, eds. G. Cayrel de Strobel, \& M. Spite, IAU Symp., 132, 253

Willamo, T., Hackman, T., Lehtinen, J. J., et al. 2019, A\&A, 622, A170 


\section{Appendix A: Observed and modelled spectra}
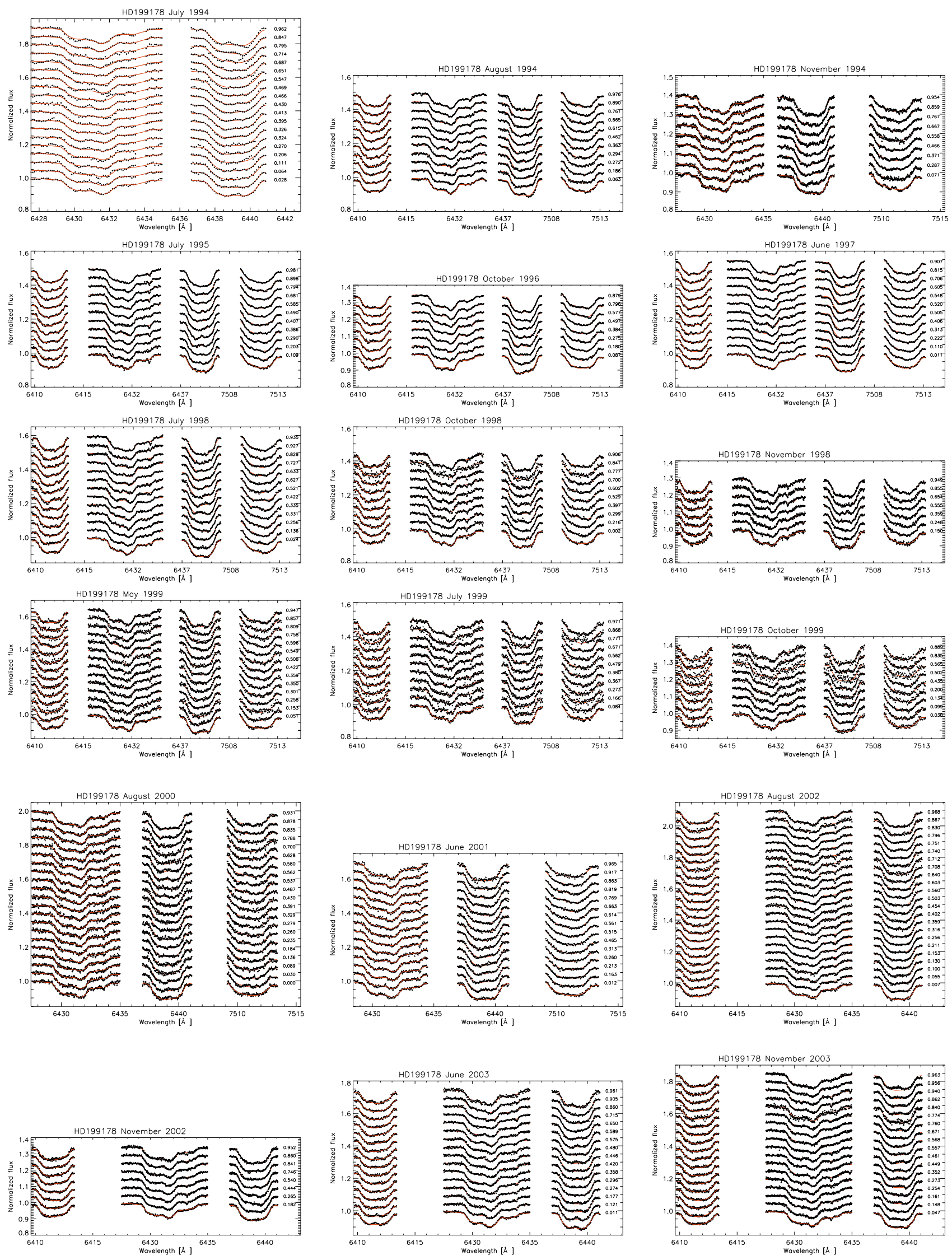

Fig. A.1. Observed (plus signs) and modelled (red line) spectra. Different wavelength regions are plotted in the same frames, the wavelength step being constant. The rotational phase is given to the right of each spectrum. 
A\&A 625, A79 (2019)
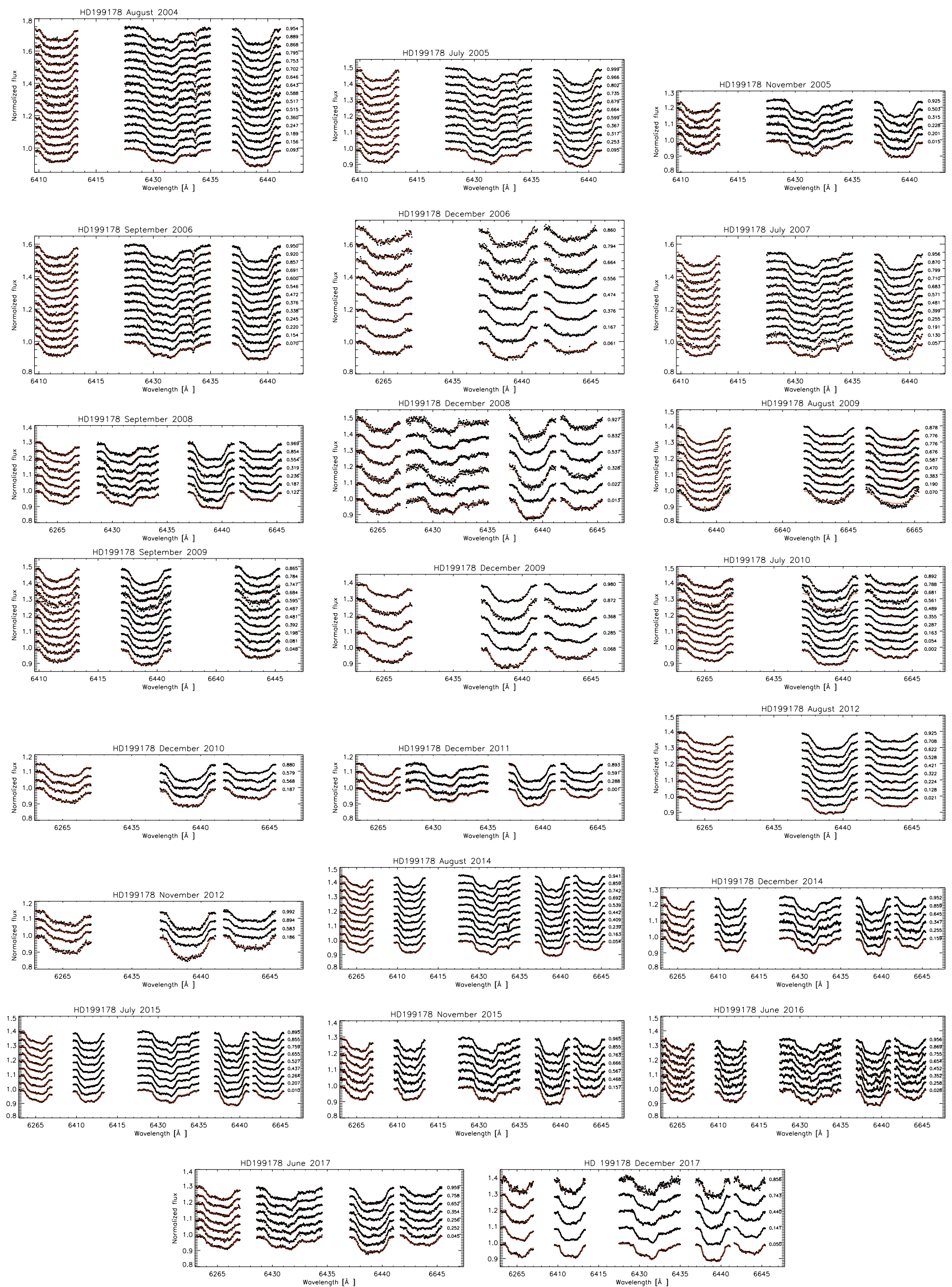

Fig. A.1. continued. 